\title{
Assessment of conventional tomography and fibreoptic bronchoscopy in the diagnosis of potentially malignant chest opacities
}

\author{
LR BAGG, ID COX, NJ RUSSELL, ANNA SC THORNTON, B GORMAN, MJ TURNER \\ From the Departments of Respiratory Medicine and Radiology, The London Hospital, London
}

ABSTRACT Conventional chest tomography and fibreoptic bronchoscopy were performed in 100 patients with a localised chest abnormality on their chest radiographs who had been referred with a possible diagnosis of bronchial carcinoma. Carcinoma was eventually confirmed in 74 cases and in 26 the lesion proved to be benign. The accuracy of tomography in the overall series was $83 \%$, with a sensitivity of $92 \%$ and a specificity of $58 \%$. These figures compare favourably with the results of other imaging techniques used in the diagnosis of bronchial carcinoma. In 50 patients bronchoscopy did not suggest carcinoma and in this group of patients 24 had a carcinoma and 26 a benign lesion. The accuracy of tomography in the bronchoscopy negative patients was $74 \%$, the major source of error being the false positive category. The lesion eventually proved to be benign in $33 \%$ of the cases where tomography suggested malignancy, but when the tomograms suggested that the lesion was benign a tumour was found to be present in only $12 \%$ of cases.

When an abnormal chest radiograph suggests the possibility of bronchial carcinoma, a sequence of investigations follows that usually includes examination of sputum for neoplastic cells and, if this gives negative results, a fibreoptic bronchoscopy. Should the result of bronchoscopy also prove negative further information about the lesion may be sought by percutaneous needle biopsy, computed tomography, gallium 67 scanning, and conventional tomography. Of these investigations, computed tomography and gallium 67 scanning are limited in their availability and needle biopsy may be inappropriate, either because of the size or site of the lesion or because the necessary expertise is not available. In many such cases tomography may therefore be the next step in the diagnostic pathway, and may critically affect management decisions. ${ }^{1-3}$

The diagnostic accuracy of the combination of fibreoptic bronchoscopy and tomography has not previously been reported. We have therefore studied the relative and combined diagnostic value of these investigations in cases where the possibility of carcinoma has been raised as a result of shadowing

Address for reprint requests: Dr LR Bagg, King George Hospital, Eastern Avenue, Ilford, Essex IG2 7RL.

Accepted 14 October 1983 on the chest radiograph. The patients who pose the most difficult problems are, of course, those in whom bronchoscopy gives a negative result. We were therefore particularly interested in clarifying the role of tomography in the management of such patients.

\section{Methods}

The patients we studied all had a localised abnormality on their chest radiograph and were referred for bronchoscopy to confirm or exclude a diagnosis of carcinoma. The series reported here consists of 100 such patients, seen consecutively, in whom bronchoscopy was performed and in whom the tomograms obtained were technically satisfactory. There were 84 men and 16 women, with an average age of 63 years.

Fibreoptic bronchoscopy was performed in a standard fashion with topical lignocaine anaesthesia induced one hour after premedication with papaveretum and hyoscine. Biopsy specimens and brushings for cytological examination were taken from visible lesions, and washings were examined for neoplastic cells in every case. A bronchoscopy was designated as giving a positive result only if histological or cytological proof was obtained from the procedure. In five instances the diagnosis was not 
Table 1 Criteria for malignancy used in assessing radiological mass lesions (listed in descending order of the weightings accorded)

1 Local or metastatic rib destruction

2 Bronchostenosis or deformity of trachea, carina, main bronchi, or proximal segmental bronchi

3 Cavitation in mass with thick irregular walls

4 Spiculation of irregular margin of mass

5 Mass with hilar enlargement

6 Lobulation of mass

7 Lobar or segmental collapse with any of the above

8 Mass accompanied by linear shadows characteristic of lymphatic infiltration

confirmed, despite appearances of extrinsic compression and distortion of the bronchial tree; these cases were categorised as bronchoscopy negative for the purposes of this study.

Tomograms were obtained at times ranging from two weeks before to two weeks after the bronchoscopy. Rectilinear tomography was performed in a longitudinal plane. In most cases oblique $55^{\circ}$ hilar projections were used, supplemented where necessary by anteroposterior and lateral sections. The tomograms were reviewed by three radiologists and categorised as positive (carcinoma) or negative (benign). The criteria of malignancy used in assessing radiological mass lesions are given in table 1 and are ranked in order of the weighting given to each feature. Combinations of two or more positive signs were regarded as strengthening the diagnosis proportionately. The radiographs and tomograms were reviewed by the three radiologists together; each gave individual opinions and the majority view was accepted. Interpretation was made without access to any information other than that inherent in the tomograms and posteroanterior and lateral chest radiographs. This placed the radiologists in a position of less advantage than they would have had in normal clinical practice but it ensured that the opinion was determined solely by the radiographic features. In 90 cases the decision was unanimous and in 10 it was based on a majority verdict. In seven of the 10 disputed cases the majority verdict was subsequently found to be correct and in three cases incorrect.

For a final diagnosis of carcinoma a positive result was required from histological or cytological examination. Diagnosis of the benign lesions was based variously on histological or microbiological examination, resolution of the abnormality on the chest radiograph, or failure to progress over the subsequent two years.

\section{Results}

Of the 100 patients, 74 eventually proved to have carcinoma and 26 had benign lesions (table 2). The
Table 2 Final diagnosis in 100 patients with a localised chest radiographic lesion referred for fibreoptic bronchoscopy

\begin{tabular}{lrlr}
\hline Benign & & Malignant \\
\hline Inflammatory lesion & 14 & Squamous cell carcinoma & 42 \\
Scarring & 8 & Adenocarcinoma & 9 \\
Sarcoidosis & 1 & Small cell carcinoma & 12 \\
Vascular anomaly & 1 & Large cell undifferentiated & \\
Exostosis & 1 & carcinoma & 11 \\
Hiatus hernia & 1 & Total & 74 \\
Total & 26 & & \\
\hline
\end{tabular}

latter were predominantly inflammatory conditions or scarring. The inflammatory conditions comprised a slowly resolving pneumonia in 11 cases, pyogenic lung abscess in two, and active tuberculosis in one. Scarring was thought to be due to old, inactive tuberculosis in five cases and was of uncertain cause in three.

The results for both tomography and bronchoscopy are given in table 3 with an analysis of their performance in table 4 . The sensitivity, predictive power, and accuracy of tomography were high, but the specificity was comparatively low. By contrast, bronchoscopy was relatively insensitive as a means of detecting carcinoma but, as would be expected from the classification criteria, the specificity and predictive power of a positive result were $100 \%$.

Table 3 Classification of diagnoses made by tomography and bronchoscopy in 100 patients with a localised radiographic lesion

\begin{tabular}{lcc}
\hline & Tomography & Bronchoscopy \\
\hline True positive & 68 & 50 \\
True negative & 15 & 26 \\
False positive & 11 & 0 \\
False negative & 6 & 24 \\
Total & 100 & 100 \\
\hline
\end{tabular}

Table 4 Diagnostic performance of tomography and bronchoscopy in 100 patients with a radiographic mass lesion

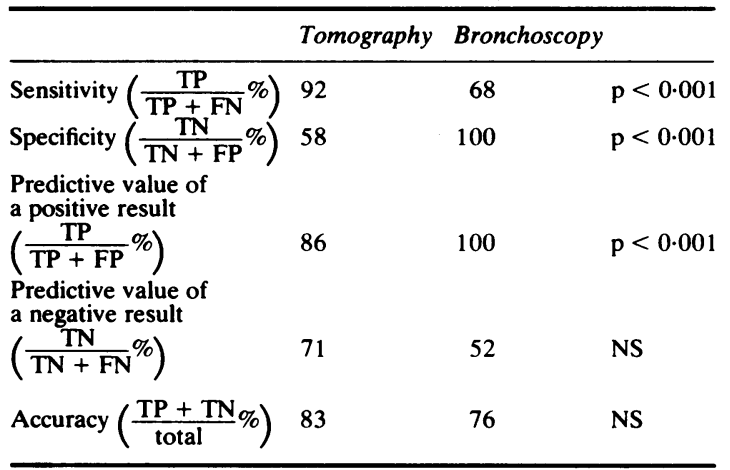

$\mathrm{TP}-$ true positive; $\mathrm{TN}-$ true negative; $\mathrm{FP}-$ false positive; $\mathrm{FN}-$ false negative; NS-not significant. 
Table 5 Diagnostic performance of tomography in 50 patients negative for carcinoma according to bronchoscopy

\begin{tabular}{|c|c|c|c|}
\hline \multicolumn{2}{|c|}{ Classification $(n=50)$} & \multicolumn{2}{|l|}{ Performance (\%) } \\
\hline $\begin{array}{l}\text { True positive } \\
\text { True negative } \\
\text { False positive }\end{array}$ & $\begin{array}{l}22 \\
15 \\
11\end{array}$ & $\begin{array}{l}\text { Sensitivity } \\
\text { Specificity } \\
\text { Predictive value of } \\
\text { a positive result }\end{array}$ & $\begin{array}{l}92 \\
58\end{array}$ \\
\hline False negative & 2 & $\begin{array}{l}\text { Predictive value of } \\
\text { a negative result } \\
\text { Accuracy }\end{array}$ & $\begin{array}{l}88 \\
74\end{array}$ \\
\hline
\end{tabular}

Bronchoscopy identified a carcinoma in four of the six false negatives yielded by tomography, but failed to establish an alternative, non-malignant cause in any of the 11 false positives. After considering information available from both tests there were therefore two cases out of the 100 with a missed diagnosis of carcinoma, and 11 lesions which ultimately proved to be benign, but in which tomography had compounded the concern that a tumour was present.

In 50 patients bronchoscopy gave a negative result, and 24 of these were eventually shown to have a carcinoma and 26 a benign lesion. Results for tomography in this group of patients are shown in table 5. The sensitivity and specificity were the same as in the overall series. The predictive value of a negative result rose to $88 \%$ since some of the false negatives were excluded by the preceding broncho- scopy. This was more than counterbalanced by the fall in predictive value of a positive result to $67 \%$, with a consequent fall in accuracy. A superficial appraisal of these changes suggests that tomography is a less accurate means of defining peripheral tumours than central lesions visualised at bronchoscopy (the accuracy fell in the bronchoscopy negative patients, except for one who had peripheral lesions outside the range of vision of the bronchoscope). In fact, the changes in test performance simply reflect the smaller proportion of carcinomas in the 50 bronchoscopy negative cases, with the false positive results forming a correspondingly increased proportion of the positive group as a whole. (The number of patients with carcinoma dropped from 74 in the overall series to 24 in the bronchoscopy negative group, but the number of false positive tomography results remained the same at 11.) The figures show that tomography was misleading in $26 \%$ of cases $(13 / 50)$, the major source of error being the false positive category. Thus one third (11/33) of the lesions diagnosed as malignant proved to be benign, whereas a tumour was present in only $12 \%$ of cases $(2 / 17)$ where the examination suggested a benign lesion.

The results are shown diagrammatically in the figure in the form of a management flow chart, giving bronchoscopy results in all 100 patients and

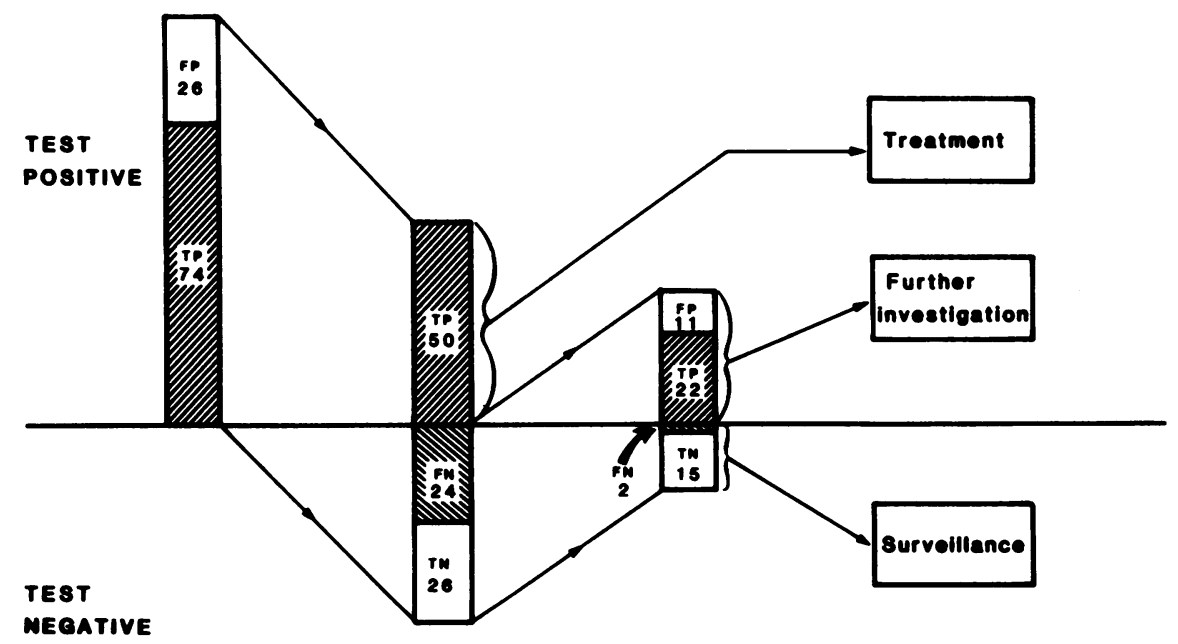

Chest Bronchoscopy Tomography
Radiograph

Results of bronchoscopy in 100 patients and of tomography in the 50 bronchoscopy negative cases, shown as a management flow chart. TP-true positive; TN-true negative; FP-false positive; $F N$-false negative. 
tomography results in the 50 bronchoscopy negative cases.

\section{Discussion}

Tomography, gallium scanning, and computed tomography are the three main imaging techniques used in the investigation of shadows on radiographs suspected of having a malignant cause. Much interest has centred on the latter two methods in recent years, and they appear to have definite advantages over conventional tomography in the assessment of mediastinal shadows and the staging of bronchial carcinoma. ${ }^{4-9}$ Computed tomography has also been shown to detect secondary deposits in the lungs which are not visible with whole lung tomography, although the clinical relevance of this is diminished by the high percentage of additional lesions so visualised which ultimately prove to be benign. ${ }^{10}$ "Where a parenchymal lesion is visible on the plain chest radiograph, and the problem lies in deciding whether it is malignant, the relative merits of the three techniques are less clear. Our figures show that in these circumstances the overall accuracy, sensitivity, and specificity of tomography are very similar to those of results reported for gallium scanning, although these vary according to how a "dubious" scan result is categorised. ${ }^{2}$ Comparable figures for computed tomography are not readily available, but Mintzer et $\mathrm{al}^{3}$ concluded that conventional tomograms were superior to computed tomography for confirming the malignant characteristics of pulmonary lesions seen on the plain radiograph. Tomography therefore appears to be at least as useful as the other two imaging techniques in the investigation of patients such as those described here and is certainly more widely available in Britain.

The accuracy of tomography at $83 \%$ is similar to that of bronchoscopy at $76 \%$. Tomography does not, however, provide a tissue diagnosis, and is very unlikely to be used as the sole basis for decisions on clinical management. Thus, given the low complication rate of fibreoptic bronchoscopy, it is improbable that the tomography result would deflect the clinician from requesting bronchoscopy if this seemed to be indicated by the original chest radiograph. The correct timing of tomography would therefore seem to be after bronchoscopy where the diagnosis remains in doubt.

When a positive diagnosis of carcinoma is made by bronchoscopy, as was the case in half our patients, tomography generally provides no further diagnostic information, although it may have some role in determining spread and the operability of the tumour. It is in the bronchoscopy negative cases that management problems arise and where the accuracy of tomography is of most interest. In a sense this is a misapplication of tomography, which because of its high sensitivity and comparatively low specificity is of more value as a screening test than as a method of establishing the diagnosis of carcinoma. Indeed, it cannot establish the diagnosis in the accepted sense of the word, but can provide only a statistical framework of probabilities. Nevertheless, in these circumstances any additional information is welcome, and our figures offer a guide to how reliable this information is. In addition, tomography is a useful prelude to needle biopsy when this is necessary.

Where bronchoscopy gives a negative result and the lesion appears benign on tomograms most clinicians would probably defer invasive investigation and confine their activities to a period of observation. In $12 \%$ of our patients in this category the lesion eventually proved to be malignant, emphasising that an expectant policy should be critically reviewed at frequent intervals. Where bronchoscopy gives a negative result but the lesion appears malignant on tomograms the possible options include thoracotomy and further investigation. In one third of our patients in this category the lesion was benign, suggesting that immediate referral for thoracotomy may be inappropriate, and that a period of further intensive investigation and exploration of other diagnostic possibilities is well justified.

\section{References}

' Miller WE, Crowe JK, Muhm JR. The evaluation of pulmonary parenchymal abnormalities by tomography. Radiol Clin North Am 1976;14:85-93.

${ }^{2}$ McLeod RA, Brown LR, Miller WE, DeRemee RA. Evaluation of the pulmonary hila by tomography. Radiol Clin North Am 1976;14:51-84.

${ }^{3}$ Mintzer RA, Malave SR, Neiman HL, Michaelis LL, Vanecko RM, Sanders JH. Computed vs conventional tomography in evaluation of primary and secondary pulmonary neoplasms. Radiology 1979;132:653-9.

${ }_{4}$ Underwood GH, Hooper RG, Axelbaum SP, Goodwin DW. Computed tomographic scanning of the thorax in the staging of bronchogenic carcinoma. $N$ Engl J Med 1979;300:777-8.

${ }^{5}$ Fosburg RG, Hopkins GB, Kan MK. Evaluation of the mediastinum by gallium-67 scintigraphy in lung cancer. J Thorac Cardiovasc Surg 1979;77:76-82.

- Robbins AH, Pugatch RD, Gerzof SG, Faling LJ, Johnson WC, Sewell DH. Observations on the medical efficacy of computed tomography of the chest and abdomen. Am J Roentgenol 1978;131:15-9.

7 Crowe JK, Brown LR, Muhm JR. Computed tomography of the mediastinum. Radiology 1978;128:7587.

${ }^{8}$ Siemsen JK, Grebe SF, Sargent EN, Wentz D. Gallium-67 scintigraphy of pulmonary diseases as a 
complement to radiography. Radiology 1976;118:371-5.

9 Goldstraw P, Kurzer M, Edwards D. Preoperative staging of lung cancer: accuracy of computed tomography versus mediastinoscopy. Thorax 1983;38:10-5.

${ }^{10}$ Sindelar WF, Bagley DH, Felix EL, Doppman JL, Ketcham AS. Lung tomography in cancer patients. Full-lung tomograms in screening for pulmonary metastases. JAMA 1978;240:2060-3.
"Schaner EG, Chang AE, Doppman JL, Conkle DM, Flye MW, Rosenberg SA. Comparison of computed and conventional whole lung tomography in detecting pulmonary nodules: a prospective radiologicpathologic study. Am J Roentgenol 1978;131:51-4.

12 Pannier R, Verlinde I, Puspowidjono I, Willemot JP. Role of gallium 67 thoracic scintigraphy in the diagnosis and staging of patients suspected of bronchial carcinoma. Thorax 1982;37:264-9. 